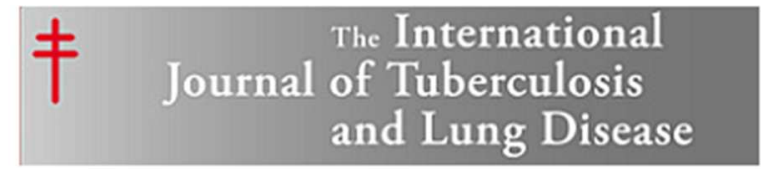

\title{
Programmatic management of patients with pre-extensively drug-resistant tuberculosis in Peru, 2011-2014
}

\begin{tabular}{|r|l|}
\hline Journal: & The International Journal of Tuberculosis and Lung Disease \\
\hline Manuscript ID & IJTLD-12-17-0900.R1 \\
\hline Manuscript Type: & Original Article \\
\hline Date Submitted by the Author: & $29-$ Apr-2018 \\
\hline Complete List of Authors: & $\begin{array}{l}\text { Alarcon, Antonieta; Ministerio de Salud, Estrategia Sanitaria Nacional de } \\
\text { Prevención y Control de la Tuberculosis } \\
\text { Alarcon, Edith; Pan-American Health Organisation, US office } \\
\text { Mendoza-Ticona, Alberto; Hospital de Emergencias Villa El Salvador, } \\
\text { Infectious Diseases Division } \\
\text { Obregon, George; Instituto Nacional de Salud, Laboratorio de Referencia } \\
\text { Nacional de Micobacterias } \\
\text { Cornejo, José; Hospital Nacional Arzobispo Loayza, Service of Neumology } \\
\text { Vargas, Dante; Hospital Nacional Hipolito Unanue, Department of Medicine } \\
\text { de los Ríos, Jorge; Hospital Maria Auxiliadora, Service of Neumology } \\
\text { Moore, David; The London School of Hygiene and Tropical Diseases, } \\
\text { Department of Clinical Research } \\
\text { Heldal, Einar; Nasjonalt folkehelseinstitutt, Public Health }\end{array}$ \\
\hline Key Words: & \begin{tabular}{l} 
pre-XDR TB, culture conversion, treatment outcome, Peru \\
\hline
\end{tabular} \\
\hline
\end{tabular}


1 2

3

\title{
Programmatic management of patients with pre-extensively drug- resistant tuberculosis in Peru, 2011-2014
}

\author{
Valentina Alarcón Guizado ${ }^{1}$, Edith Alarcón Arrascue ${ }^{2}$, Alberto Mendoza-Ticona ${ }^{1,3}$, George Obregón Boltan ${ }^{4}$, José \\ Cornejo García $^{1,5}$, Dante Vargas ${ }^{1,6}$, Jorge de los Ríos ${ }^{1,7}$, David AJ Moore ${ }^{8}$ and Einar Heldal ${ }^{9}$ \\ 1: Estrategia Sanitaria Nacional de Prevención y Control de la Tuberculosis, Ministerio de Salud, Peru \\ 2: The International Union against Tuberculosis and Lung Disease, Peru \\ 3: Hospital de Emergencias Villa El Salvador, Ministerio de Salud, Peru \\ 4: Instituto Nacional de Salud de Perú \\ 5: Hospital Nacional Arzobispo Loayza, Ministerio de Salud, Peru \\ 6: Hospital Nacional Hipólito Unanue, Ministerio de Salud, Peru \\ 7: Hospital María Auxiliadora, Ministerio de Salud, Peru \\ 8: The London School of Hygiene and Tropical Diseases, UK \\ 9: The International Union against Tuberculosis and Lung Disease, Norway \\ Running head: Pre-extensively drug-resistant tuberculosis in Peru \\ Summary: 200 (Max 200) \\ Text: 2495 (Max 2500) \\ Number of references: 20 \\ Number of tables and figures: 6 \\ Keywords: Pre-XDR TB, culture conversion, treatment outcome, Peru

\section{Corresponding author: Valentina Alarcón Guizado} \\ Address: Av. Cesar Vallejo, cuadra $13 \mathrm{~s} / \mathrm{n}$, El Agustino \\ Telephone: +51-99079882; +5113620765 \\ Email: antonieta_alarcong@hotmail.com
}


31 ABSTRACT:

32 Background: In Peru, a treatment approach for XDR-TB that incorporated WHO group

335 drugs and a patient-centered care, has achieved $65 \%$ success. To expand this 34 approach for pre-XDR-TB, we have evaluated this population separately.

35 Objective: To assess programmatic management of pre-XDR-TB.

36 Method: Retrospective study using official registry from 2011 to 2014. Cases were 37 separately evaluated according to resistance to fluoroquinolones (pre-XDR-F) or $2^{\text {nd }}$ 38 line injectable drugs (SLIs) (pre-XDR-I).

39 Results: From 610 pre-XDR-TB patients, 120 (20\%) had pre-XDR-F and 490 (80\%) had 40 pre-XDR-I. The pre-XDR-F cases were older (34 vs 28 years, $p<0,001$ ) and a higher 41 proportion had previously received two or more regimens $(70 \%$ vs $38 \%, p<0,001)$. In 42 the 452 cases who started treatment in 2011-2013, treatment success was $43.3 \%$, $4326.5 \%$ were lost to follow-up, $12.1 \%$ died and $13.7 \%$ failed treatment. Success was 44 higher in pre-XDR-I (48.5\%) than pre-XDR-F (21.4\%). History of previous treatment (OR $452.23, \mathrm{Cl} 1.52-3.38)$ and pre-XDR-F (OR 2.39, Cl 1.18 - 4.83) were associated with 46 unsuccessful outcome.

47 Conclusion: Programmatic management of pre-XDR-TB has not been successful, 48 especially in pre-XDR-F, with lower rates of success than have been achieved in the 49 same setting for XDR-TB. The strategy used for XDR-TB should be expanded to pre$50 \mathrm{XDR}-\mathrm{TB}$ in Peru. 
Drug-resistant tuberculosis (TB) poses a challenge for TB elimination, with 660,000 estimated new cases resistant to rifampicin (RR-TB), of which 490,000 had multidrugresistant TB (MDR-TB) with resistance to at least isoniazid $(H)$ and rifampicin $(R)$ in 2016 worldwide. ${ }^{1}$ Among MDR-TB cases, 6.2\% fulfil the criteria for XDR-TB with additional resistance to a fluoroquinolone and a second line injectable drug (SLI) [kanamycin $(\mathrm{Km})$, capreomycin $(\mathrm{Cm})$ or amikacin $(\mathrm{Am})] .{ }^{1}$ Pre-XDR-TB is defined as MDR-TB with resistance to either a fluoroquinolone (pre-XDR-F) or a SLI (pre-XDR-I), but not both. ${ }^{2}$ The burden of pre-XDR-TB is considerable and represents a threat to MDR-TB control; the proportion of MDR-TB with resistance to any fluoroquinolone was estimated at $21 \%$ worldwide in 2015 , with an overall $51 \%$ with resistance to a fluoroquinolone or a SLI. ${ }^{3}$

In 2015, Peru reported 1,366 cases of MDR-TB and 104 cases of XDR-TB. ${ }^{4}$ Since 2011, Peru has provided individualized treatment for MDR-TB based on drug susceptibility testing (DST) of first and second-line agents, as per WHO recommendations, with at least 4 effective drugs delivered through the primary health care system ${ }^{5}$. MDR-TB treatment success rate in 2013 was 55\%, with 29\% of patients lost to follow-up, data that included pre-XDR TB. During the same period, a more aggressive and multifaceted approach to the treatment of XDR-TB entailed: i) the additional use of drugs from Group 5 of the former WHO classification and thioridazine (not used for non-XDR MDR-TB), ii) a comprehensive care package starting in hospital, with follow-up at home and ending in primary care, iii) direct observation of treatment, iv) additional social support, v) improved monthly food baskets and vi) infection control measures in the home. ${ }^{4}$ With this package of interventions, the treatment success for XDR-TB rose from 30\% in 2011 to 66\% in 2013 (higher than for programmatic management of MDRTB), while loss to follow-up decreased from $27 \%$ to $2 \%{ }^{6}$

Resistance to fluoroquinolones and SLIs reduces the success rate for MDR-TB therapy, and though there are a growing number of studies on XDR-TB treatment ${ }^{7-9}$ there is a scarcity of data on the much more common pre-XDR-TB. ${ }^{2,10}$ The National Tuberculosis Programme (NTP) in Peru suspected that the low success rate in MDR-TB might be due to poor treatment outcomes amongst pre-XDR-TB cases, which are normally included 
82 in the MDR-TB cohort. The objective of this study was to assess retrospectively the 83 programmatic management of pre-XDR-TB cases who started treatment between 2011 84 and 2014, evaluate time between diagnosis and treatment initiation, drugs used, 85 culture conversion and treatment outcome among those who started treatment in 86 2011-2013, disaggregated into cases with pre-XDR-F and pre-XDR-I. 


\section{METHODS}

The operational study to compare retrospective cohorts of pre-XDR-TB cases.

Context: Since 2013, the NTP has required DST to $\mathrm{R}$ and $\mathrm{H}$ for all diagnosed TB cases in the country using direct rapid assays validated in the country, either nitrate reductase (Griess) assay ${ }^{11}$, direct microscopic-observation drug-susceptibility (MODS) assay ${ }^{12,13}$ or molecular line probe assay (LPA). ${ }^{14}$. On detection of $H$ or $\mathrm{R}$ resistance, indirect culture-based DST for first and second line drugs using Middlebrook 7H10 agar plates proportion (APP) assay is performed at the National Mycobacterial Reference Laboratory, with annual external quality evaluation by the Supranational Laboratory of WHO. Among fluoroquinolones, ciprofloxacin was tested, and for SLI kanamycin ( Km) and capreomycin $(\mathrm{Cm})$. Before and even after the 2013 national standard was issued, DST continued to be performed using the indirect proportion method in APP or Lowenstein Jensen (LJ) medium. Due to delays in results of indirect DST, many patients started standardized treatment with second-line drugs after having failed first-line treatment, or because they were contacts of MDR-TB, before obtaining indirect DST results.

Evaluation Committees on Retreatment approved regimens for MDR-TB cases, and treatment was administered on an outpatient basis at the first level of care, at the clinic nearest to the patient's home. MDR-TB regimens based on rapid $H$ and $R$ assays were later adjusted to individualized regimens when the APP became available, according to Peruvian guidelines ${ }^{15,16}$. In some cases, the regimen was maintained if the clinical course was favorable. The best available drugs were selected, based on the previous WHO classification into five groups, including at least four effective drugs ${ }^{5}$. In cases of pre-XDR-TB, a fluoroquinolone or an SLI was included; in case of fluoroquinolone resistance, moxifloxacin (Mfx) was added, and in case of SLI resistance, $\mathrm{Cm}$ or amikacin (Am) were added, in both cases these drugs were not counted as one of the four required effective drugs. Ethionamide (Eto), cycloserine (Cs) and ethambutol (E) were added if the strain was still susceptible to these drugs, usually maintaining pyrazinamide $(\mathrm{Z})$. In cases with resistance to Eto, the drugs $E, Z$ and/or para-aminosalicylic acid (PAS) were always added with the goal to get four effective drugs. 
118 The elevated cost of group 5 drugs plus hospitalization, insertion of a central line for 119 carbapenems, and DOT in the household had a high cost for the government; these 120 drugs were therefore limited to XDR-TB cases. For pre-XDR-TB a supposedly adequate 121 individualized regimen could be designed based on either a fluoroquinolone or a SLI, 122 plus Eto, E, Z, Amx-Clv, Cs and PAS in line with WHO guidelines of 2011. ${ }^{5}$ Confirmed cases of XDR-TB were treated also with linezolid (Lzd), carbapenems plus amoxicillin clavulanate (Amx-Clv), as well as thioridazine ${ }^{4}$. Bacteriological conversion was monitored by monthly sputum cultures. ${ }^{15}$

Study population: Pulmonary pre-XDR-TB patients who started treatment from January 2011 to December 2014 were included. Patients were classified in two cohorts based on fluoroquinolone (ciprofloxacin) resistance (pre-XDR-F TB), or resistance to one or both SLIs (Km, Cm) (pre-XDR-I TB), as assessed by APP. Patients of all ages and from all over the country were included. For treatment outcome, the subgroup of patients who started treatment in 2011-2013 was assessed. Treatment outcomes were: cured, treatment completed, failure, lost to follow-up, deceased, and not evaluated, in accordance with WHO Guidelines. ${ }^{17}$ Two authors examined the clinical records of all patients to determine treatment outcome. For evaluating monthly culture results and treatment outcome, the group of pre-XDR-I was subdivided in three: pre-XDR-Km, pre-XDR-Cm and pre-XDR-Km-Cm.

Data collection: The National Resistant Tuberculosis Registry was used, which is updated daily with quarterly reports of each resistant TB case in the country. The result of the DST was checked with the computerized NETLAB database. ${ }^{18}$ Demographic variables, treatment history, comorbidities, monthly culture results, DST results, treatment regimens, and outcomes were evaluated in accordance with WHO guidelines. The outcomes were re-categorized in successful (cure and completed treatment) and unsuccessful (failure, death, lost to follow-up and not evaluated).

Statistical analysis: The database was analyzed using STATA version 14.2 (Stata corp, 146 Texas, USA). Clinical and epidemiological characteristics were compared between pre147 XDR-F and pre-XDR-I patients. A Chi-squared test $\left(X^{2}\right)$ was used to assess the association between categorical variables, while a Wilcoxon test was used for 
149 continuous variables. In the subgroup of patients registered in 2011-2013, treatment 150 outcome was compared between pre-XDR-F and pre-XDR-I cases and injectable 151 subgroups. A p-value $<0.05$ was considered to define a significant difference. 152 Multivariate analysis was performed to identify factors significantly associated with 153 unsuccessful treatment outcome.

154 Ethical considerations: The protocol was approved by the Ethics Committees at the 155 Hospital Nacional Hipólito Unanue, and from The Union. 
A total of 610 pre-XDR-TB patients who started treatment in 2011-2014 were included: 120 (19.7\%) with pre-XDR-F and 490 (80.3\%) with pre-XDR-I. Among pre-XDR-I, 213 (43.5\%) were resistant to $\mathrm{Km}$ only, 102 (20.8\%) were resistant to $\mathrm{Cm}$ only, and 175 (35.7\%) were resistant to both injectable drugs.

Male gender was more common in both cohorts, median age was 29 years old. Diabetes mellitus was recorded for $8 \%$ of patients and HIV in $4 \%$. Among pre-XDR-TB patients, $67 \%$ had undergone two or more previous courses of TB treatment. Pre-XDRF patients were significantly older (median 34 vs 28 years, $p<0.001$ ), more commonly reported both diabetes and previous treatment episodes: $70 \%$ had received two or more prior courses of treatment vs. $38 \%$ of pre-XDR-I patients $(p<0.001)$ and most of them received their treatment after previous loss to follow-up (Table 1).

Treatment for drug-resistant TB was started in $29 \%$ of patients pending bacteriological confirmation with an indirect DST. Treatment was started in $39 \%$ of patients less than two weeks after diagnosis, and 32\% after more than 3 weeks, with no significant differences between the groups (Table 2).

The drugs from the individualized regimens that were used in more than $50 \%$ of the patients, were grouped based on the resistance pattern: i) for pre-XDR-F: Cm, Z, Mfx, Cs, Eto and Amx-Clv, ii) for pre-XDR-I Km-resistant: Z, Cm, Lfx, PAS, Cs and Eto, iii) for pre-XDR-I Cm-resistant: Z, Lfx, PAS, Cs, Eto and Amx-Clv and iv) for pre-XDR-I Km+Cmresistant: Z, Lfx, PAS, Cs, Eto and Amx-Clv. Drugs from previous Group 5 and thioridazine were very rarely used (Table 3 ).

Of the 610 patients, 342 (56\%) had achieved bacteriological conversion by the sixth month; this was higher in the pre-XDR-I group (60.6\%) than in pre-XDR-F group $(42.5 \%), p<0.001$. The proportion with positive culture by month of treatment was higher in the pre-XDR-F group, followed by the pre-XDR $\mathrm{Km}+\mathrm{Cm}$ (Figure 1). The number not assessed for conversion (because of death, loss to follow-up or missing information) were in total 157 (26\%): 41 (34\%) pre-XDR-F, 53 (25\%) pre-XDR-Km, 25 (25\%) pre-XDR-Cm and 38 (22\%) pre-XDR-Km+Cm. 
185 Among the 452 patients who started treatment in $2011-2013,43.4 \%$ were successfully 186 treated, $26.5 \%$ were lost to follow-up, $13.7 \%$ had treatment failure, $12.2 \%$ were 187 deceased, and in $4.2 \%$ treatment outcome was not evaluated. A successful treatment 188 outcome was significantly less likely in patients with pre-XDR-F (21.4\%) vs. pre-XDR-I 189 (48.5\%), $p<0.001$. Among pre-XDR-I patients, the lowest success rate was seen in 190 patients with resistance to both $\mathrm{Km}$ and $\mathrm{Cm}$ (Table 4). Among patients with lost to 191 follow result, $48 \%$ occurred during first to six month, 34\% at seven to 12 month, and $19218 \%$ after 12 months. Treatment failure and death rates were lower in pre-XDR-I with $193 \mathrm{Cm}$ resistance. The highest proportion of death (22.6\%) was in the pre-XDR-F type. In a 194 multivariate model, the only variables independently associated with unsuccessful 195 outcome were a history of previous treatment and resistance to fluoroquinolones (pre196 XDR-F), OR 2.23 (1.52 - 3.38), p<0.001 and OR 2.39 (1.18 - 4.83), $p<0.015$, respectively 197 (Table 5). 
200 The study is one of few published on programmatic management of pre-XDR-TB and

201 the first nationwide study in Peru. The overall success rate in pre-XDR was low, even 202 surprisingly lower than XDR-TB but this reflects the intensive attentio ${ }^{2} n$ paid to 203 treating XDR-TB patients in Peru, with particularly strengthened treatment regimens, 204 patient-centralized DOT, and social support. ${ }^{4}$ The study confirmed significantly lower 205 treatment success in pre-XDR-TB with resistance to fluoroquinolones than to SLI, as 206 also found in previous reports. ${ }^{2,10}$

207 Even though pre-XDR-TB patients were diagnosed quickly and treated in line with WHO 208 recommendations, ${ }^{5}$ the majority of cases had unfavorable outcomes. The most 209 frequent unfavorable outcome was lost to follow up with $26.4 \%$, which were more 210 frequent in the first six months, and probably due to long and weak treatment 211 regimens, clinic-centered DOT, adverse reactions, inadequate social support, and 212 limitations of the health system to follow up timely patients who take their treatment 213 irregularly. The high death rate was likely due in the early years to a delay in DST 214 results for second-line drugs (with conventional method) and late treatment initiation, 215 although it subsequently remained high even though rapid DST coverage was 216 increased. Comorbidities or coinfections may have contributed to the increased 217 mortality as well. The death rate was higher in pre-XDR-F than pre-XDR-I, possibly 218 because these patients were older and had received more previous treatments. 219 Acquired resistance may explain the high failure rate as patient already with pre-XDR 220 were started on standardized MDR-TB treatment because DST results came late. 221 Fluoroquinolone resistance was only tested to ciprofloxacin. Probably a considerable proportion of strains with resistance to ciprofloxacin maintained susceptibility to 223 levofloxacin or moxifloxacin with which they were treated, but still ciprofloxacin 224 resistance was associated with poor outcome in a multivariable model. There was no DST for Am so the use of this injectable may not have been optimal.

226 Our study is unusual in finding higher success rate in XDR-TB than pre-XDR-TB; lower 227 treatment success rate in pre-XDR-F than pre-XDR-I has also been found in other 228 studies. Data from 6724 MDR-TB patients with personalized treatment in 26 sites 229 worldwide showed a $64 \%$ success rate in cases with no resistance to injectable or 
230 fluoroquinolones, $56 \%$ in pre-XDR-I, $48 \%$ in pre-XDR-F and $40 \%$ in XDR. Failure/relapse 231 rate increased with increasing resistance (from $4 \%$ in MDR-TB to $22 \%$ in XDR-TB), as 232 did deaths (from 8 to 15\%), but not the proportion lost to follow-up (18-16-12-16\%, 233 respectively) ${ }^{10}$.In 1407 MDR-TB patients from Korea, similar data were observed, with 234 a $47 \%$ success rate in cases with no fluoroquinolone and injectable resistance, the same success rate (47\%) in patients with pre-XDR-I, but lower in pre-XDR-F (36\%) and 236 in XDR (29\%). Failure and death rates increased with increased resistance, while 237 patients lost to follow-up decreased with increasing resistance. ${ }^{2}$

A strength was that the study was nation-wide, with DST done centralized in an externally quality assured reference laboratory. One limitation was that fluoroquinolone susceptibility was only tested for ciprofloxacin, while treatment included levofloxacin or moxifloxacin. Another limitation was incomplete culture conversion data because data were missing or many patients lost or died. Instead, the proportion of all cases with positive culture by month was shown for each subgroup, but such grouped data must be interpreted with caution. Recording of adverse drug reactions was incomplete at central level, so that the relationship with loss to followup could not be assessed.

The recommendations arising from our findings are that: i) pre-XDR-TB cases should be handled similarly to XDR TB cases, incorporating new effective drugs (current WHO Groups C \& D) with special care for patients resistant to fluoroquinolones, ii) all MDRTB patients (and not just XDR-TB patients) need patient-centered strategies to prevent and reduce loss to follow-up, with strictly supervised home treatment (intensive phase) and then as outpatients at the health facility (continuation phase), iii) rapid DST for fluoroquinolone and SLI should be implemented in MDR-TB high burden countries, iv) surveillance for adverse events should be improved, and v) patient cohort outcomes should be evaluated separately for MDR-TB, pre-XDR-F TB, pre-XDR-I TB and XDR-TB.

Our results suggest that the current definition of pre-XDR-TB as a distinct entity may not be a very useful concept since it consists of two very different groups with different treatment success rates. As of January 2016, pre-XDR-TB patients have been managed under the same conditions as XDR-TB patients in Peru and in line with other reports ${ }^{19}$ and WHO guidelines from $2016 .{ }^{20}$ 
261 In conclusion, previous programmatic management of pre-XDR-TB, treated as other

262 MDR-TB patients, had too low success rate, especially in cases of pre-XDR-F. Rapid 263 drug susceptibility testing for fluoroquinolones and SLIs, patient-centered strategies 264 and regimens including new drugs are needed.

265 


\section{ACKNOWLEDGEMENTS}

267 This research was made possible with support from the United States Agency for 268 International Development (USAID) through the TREAT TB Cooperative Agreement 269 (AID-GHN-A-00-08-00004). The contents are the responsibility of the authors and do 270 not necessarily reflect the views of USAID or the United States Government. Additional 271 support was provided through the Structured Operational Research and Training 272 Initiative (SORT IT), a global partnership led by UNICEF/UNDP/World Bank/WHO 273 Special Programme for Research and Training in Tropical Diseases (TDR) based at the 274 World Health Organization. 
REFERENCES:

1. World Health Organization. Global Tuberculosis Report 2017. Geneva: Licence: CC BYNCSA 3.0 IGO. WHO/HTM/TB/2017.23 2017.

2. Kim DH, Kim HJ, Park SK, et al. Treatment outcomes and survival based on drug resistance patterns in multidrug-resistant tuberculosis. American journal of respiratory and critical care medicine. 2010;182(1):113-119.

3. World Health Organization. Global Tuberculosis Report 2016. Geneva: WHO/HTM/TB/2016.132016.

4. Alarcon V, Alarcon E, Figueroa C, et al. [Tuberculosis in Peru: epidemiological situation, progress and challenges for its control]. Revista peruana de medicina experimental y salud publica. 2017;34(2):299-310.

5. World Health Organization. Guidelines for the programmatic management of drugresistant tuberculosis - 2011 update. WHO Document Production Services, Geneva, Switzerland. WHO/HTM/TB/2011.6 2011.

6. Alarcon A. La experiencia en el manejo programático de la TB-XDR en Perú Available: http://www.tuberculosis.minsa.gob.pe/Archivos/Recursolnformacion/201609200407.pdf.

7. Johnston JC, Shahidi NC, Sadatsafavi M, et al. Treatment outcomes of multidrugresistant tuberculosis: a systematic review and meta-analysis. PloS one. 2009;4(9):e6914.

8. Orenstein EW, Basu S, Shah NS, et al. Treatment outcomes among patients with multidrug-resistant tuberculosis: systematic review and meta-analysis. The Lancet Infectious diseases. 2009;9(3):153-161.

9. Javaid A, Ullah I, Masud $\mathrm{H}$, et al. Predictors of poor treatment outcomes in multidrugresistant tuberculosis patients: a retrospective cohort study. Clinical microbiology and infection : the official publication of the European Society of Clinical Microbiology and Infectious Diseases. 2017.

10. Falzon D, Gandhi N, Migliori GB, et al. Resistance to fluoroquinolones and second-line injectable drugs: impact on multidrug-resistant TB outcomes. The European respiratory journal. 2013;42(1):156-168.

11. Solis LA, Shin SS, Han LL, et al. Validation of a rapid method for detection of M. tuberculosis resistance to isoniazid and rifampin in Lima, Peru. The international journal of tuberculosis and lung disease : the official journal of the International Union against Tuberculosis and Lung Disease. 2005;9(7):760-764.

12. Caviedes L, Lee TS, Gilman RH, et al. Rapid, efficient detection and drug susceptibility testing of Mycobacterium tuberculosis in sputum by microscopic observation of broth cultures. The Tuberculosis Working Group in Peru. Journal of clinical microbiology. 2000;38(3):12031208.

13. Moore DA, Evans CA, Gilman RH, et al. Microscopic-observation drug-susceptibility assay for the diagnosis of TB. The New England journal of medicine. 2006;355(15):1539-1550.

14. Asencios L, Galarza M, Quispe N, et al. [Molecular test Genotype(R) MTBDRplus, an alternative to rapid detection of multidrug resistance tuberculosis]. Revista peruana de medicina experimental y salud publica. 2012;29(1):92-98.

15. Ministerio de Salud. Norma técnica de salud para la atención integral de las personas afectadas por tuberculosis. Lima - Perú. Available: http://www.tuberculosis.minsa.gob.pe/Archivos/norma tecnica.pdf.

16. Mendoza-Ticona A, Moore DA, Alarcon V, et al. [Proposal of anti-tuberculosis regimens based on susceptibility to isoniazid and rifampicin]. Revista peruana de medicina experimental y salud publica. 2013;30(2):197-204.

17. World Health Organization. Definiciones y marco de trabajo para la notificación de tuberculosis - revisión $2013 . \quad$ Available: 
326 http://www.paho.org/hq/index.php?option=com docman\&task=doc view\&gid=22984\&ltemi $327 \quad \mathrm{~d}=270 \&$ lang=en.

328 18. Vargas-Herrera J, Segovia-Juarez J, Garro Nunez GM. [Information system of the 329 national network of public health laboratories in Peru (Netlab)]. Revista peruana de medicina 330 experimental y salud publica. 2015;32(2):378-384.

331 19. Palmero D, Gonzalez Montaner $\mathrm{P}$, Cufre $\mathrm{M}$, et al. First series of patients with XDR and 332 pre-XDR TB treated with regimens that included meropenen-clavulanate in Argentina. Archivos 333 de bronconeumologia. 2015;51(10):e49-52.

334 20. World Health Organization. WHO Treatment guidelines for drug-resistant tuberculosis $335-2016$ update. Geneva: WHO/HTM/TB/2016.04 2016.

336

337 
Table 1. General characteristics of the study population of pre-XDR-TB patients who started treatment, Peru: 2011-2014

\begin{tabular}{|c|c|c|c|c|}
\hline \multirow[t]{2}{*}{ Characteristics } & $\begin{array}{c}\text { Pre-XDR-F } \\
(\mathrm{N}=120)\end{array}$ & $\begin{array}{c}\text { Pre-XDR-I } \\
(\mathrm{N}=490)\end{array}$ & $\begin{array}{c}\text { Total } \\
(\mathrm{N}=610)\end{array}$ & p-value \\
\hline & $n(\%)$ & n (\%) & $n(\%)$ & \\
\hline Male & $90(75)$ & $325(63)$ & $415(68)$ & 0.07 \\
\hline \multicolumn{5}{|l|}{ Age groups } \\
\hline 0 - 14 years & $1(1)$ & $5(1)$ & $6(1)$ & \\
\hline 15 - 34 years & $62(52)$ & $332(68)$ & $394(65)$ & \\
\hline 35 - 54 years & $42(35)$ & $123(25)$ & $165(27)$ & \\
\hline 55 years or more & $15(13)$ & $30(6)$ & $45(7)$ & \\
\hline Age - median (IQR) & $34(27-45)$ & $28(22-38)$ & $\begin{array}{c}29(22- \\
40)\end{array}$ & $<0.001$ \\
\hline HIV-positive & $5(4)$ & $18(4)$ & $23(4)$ & 0.71 \\
\hline Diabetes mellitus & $17(14)$ & $29(6)$ & $46(8)$ & 0.002 \\
\hline \multicolumn{5}{|l|}{ Place of origin } \\
\hline Lima and Callao & $86(72)$ & $393(80)$ & $479(79)$ & 0.041 \\
\hline \multicolumn{5}{|l|}{ Number of previous treatments } \\
\hline No previous treatments & $20(17)$ & $183(37)$ & $203(33)$ & $<0.001$ \\
\hline 1 previous treatment & $16(13)$ & $121(25)$ & $137(22)$ & \\
\hline 2 or more previous treatments & $84(70)$ & $186(38)$ & $270(44)$ & \\
\hline \multicolumn{5}{|l|}{ Treatment history } \\
\hline New & $20(17)$ & $183(37)$ & $203(33)$ & $<0.001$ \\
\hline Relapse & $12(10)$ & $53(11)$ & $65(11)$ & \\
\hline Treatment after loss to follow-up & $65(54)$ & $169(34)$ & $234(38)$ & \\
\hline Treatment after failure & $23(19)$ & $85(17)$ & $108(18)$ & \\
\hline \multicolumn{5}{|l|}{ Year of treatment initiation } \\
\hline 2011 & $33(28)$ & $133(27)$ & $166(27)$ & 0.136 \\
\hline 2012 & $19(16)$ & $125(26)$ & $144(24)$ & \\
\hline 2013 & $32(27)$ & $111(23)$ & $143(23)$ & \\
\hline 2014 & $36(30)$ & $121(25)$ & $157(26)$ & \\
\hline
\end{tabular}


Table 2. Days from diagnosis to treatment initiation for pre-XDR-TB, Peru: 20112014

\begin{tabular}{|lcccc|}
\hline Treatment initiation & Pre-XDR-F & Pre-XDR-I & Total & $\begin{array}{c}\text { p- } \\
\text { value }\end{array}$ \\
\cline { 2 - 5 } & $\mathbf{( N = 1 2 0 )}$ & $\mathbf{( N = 4 9 0 )}$ & $\mathbf{( N = 6 1 0 )}$ & \\
\hline Before diagnosis, $\mathbf{n}(\%)$ & $33(28)$ & $143(29)$ & $176(29)$ & 0.80 \\
\hline After diagnosis, $\mathbf{n}(\%)$ & $87(72)$ & $347(71)$ & $434(71)$ & \\
\hline Before diagnosis, median (IQR) & $-30(-66,-7)$ & $-22(-58,-8)$ & $-225(-61,-8)$ & 0.79 \\
\hline After diagnosis, median (IQR) & $14.5(7,28)$ & $14(6,23)$ & $14(6,24)$ & 0.23 \\
\hline After diagnosis: & & & & \\
\hline Less than 2 weeks, $\mathrm{n}(\%)$ & $50(42)$ & $187(38)$ & $237(39)$ & 0.54 \\
\hline 3-4 weeks, $\mathrm{n}(\%)$ & $20(17)$ & $101(21)$ & $121(20)$ & 0.40 \\
\hline Over 4 weeks, $\mathrm{n}(\%)$ & $17(14)$ & $59(12)$ & $76(12)$ & 0.63 \\
\hline
\end{tabular}

347 IQR: Interquartile range 
Table 3. Drugs used according to resistance pattern in pre-XDR-TB patients, Peru: 2011-2014

\begin{tabular}{|c|c|c|c|c|c|c|c|c|c|c|}
\hline \multirow{2}{*}{$\begin{array}{l}\text { Resistance } \\
\text { pattern }\end{array}$} & \multicolumn{2}{|c|}{ Pre-XDR-F } & \multicolumn{2}{|c|}{ Pre-XDR-Km } & \multicolumn{2}{|c|}{ Pre-XDR-Cm } & \multicolumn{2}{|c|}{ Pre $-\mathrm{XDR}-\mathrm{Km}+\mathrm{Cm}$} & \multicolumn{2}{|c|}{ Total } \\
\hline & $n$ & $(\%)$ & $n$ & (\%) & $\mathrm{n}$ & $(\%)$ & $\mathrm{n}$ & $(\%)$ & $n$ & (\%) \\
\hline Total & 120 & & 213 & & 102 & & 175 & & 610 & \\
\hline E & 43 & (36) & 89 & $(42)$ & 34 & (3) & 52 & (30) & 218 & (36) \\
\hline $\mathbf{z}$ & 77 & (64) & 148 & (70) & 70 & (69) & 137 & (78) & 432 & (71) \\
\hline S & 3 & (3) & 8 & (4) & 2 & $(2)$ & 16 & (9) & 29 & (5) \\
\hline $\mathrm{Km}$ & 22 & (18) & 18 & (9) & 34 & (33) & 56 & $(32)$ & 130 & (2) \\
\hline $\mathrm{Cm}$ & 57 & (48) & 164 & (77) & 5 & (5) & 71 & (41) & 297 & (49) \\
\hline Am & 22 & (18) & 18 & (9) & 34 & (33) & 56 & $(32)$ & 130 & (21) \\
\hline Cfz & 0 & (0) & 3 & (1) & 0 & $(0)$ & 1 & (1) & 4 & (1) \\
\hline Lfx & 33 & (28) & 140 & $(66)$ & 81 & (80) & 89 & $(51)$ & 343 & (56) \\
\hline Mfx & 77 & (64) & 66 & (31) & 17 & (17) & 81 & (46) & 241 & (40) \\
\hline PAS & 61 & (51) & 118 & (55) & 57 & (56) & 105 & $(60)$ & 341 & (56) \\
\hline Cs & 105 & (88) & 203 & (95) & 100 & (98) & 168 & (96) & 576 & (94) \\
\hline Thz & 4 & (3) & 3 & (1) & 0 & (0) & 6 & (3) & 13 & (2) \\
\hline Eto & 76 & (63) & 127 & $(60)$ & 83 & (81) & 98 & $(56)$ & 384 & (63) \\
\hline Lzd & 4 & (3) & 4 & (1) & 0 & (0) & 6 & (3) & 14 & (2) \\
\hline Amx-Clv & 61 & (51) & 90 & (42) & 36 & (35) & 100 & (57) & 287 & (47) \\
\hline Imp & 3 & (3) & 0 & (0) & 0 & $(0)$ & 3 & (2) & 6 & (1) \\
\hline
\end{tabular}

351 
Table 4. Clinical outcomes for pre-XDR-TB cases according to baseline resistance pattern, Peru: 2011-2013

356

\begin{tabular}{lccccccc}
\hline \multirow{2}{*}{$\begin{array}{l}\text { Treatment } \\
\text { outcomes }\end{array}$} & $\begin{array}{c}\text { Pre-XDR- } \\
\text { F }\end{array}$ & $\begin{array}{c}\text { Pre-XDR- } \\
\text { Km }\end{array}$ & $\begin{array}{c}\text { Pre-XDR- } \\
\mathbf{C m}\end{array}$ & $\begin{array}{c}\text { Pre-XDR } \\
\mathbf{K m}-\mathbf{C m}\end{array}$ & \multirow{2}{*}{ Total } & \multirow{2}{*}{ P Value } \\
\cline { 2 - 6 } & $(\mathrm{n}=83$ & $(\mathrm{n}=155)$ & $(\mathrm{n}=73)$ & $(\mathrm{n}=141)$ & $(\mathrm{n}=452)$ & \\
\hline Success & $18(21.7)$ & $81(52.3)$ & $37(50.7)$ & $60(42.6)$ & $196(43.4)$ & $<0.001$ \\
Lost to follow-up & $25(30.1)$ & $35(22.6)$ & $28(38.4)$ & $32(22.7)$ & $120(26.5)$ & 0.043 \\
Failure & $15(18.1)$ & $18(11.6)$ & $3(4.1)$ & $26(18.4)$ & $62(13.7)$ & 0.018 \\
Death & $19(22.9)$ & $16(10.3)$ & $2(2.7)$ & $18(12.8)$ & $55(12.2)$ & 0.0016 \\
Not evaluated & $6(7.2)$ & $5(3.2)$ & $3(4.1)$ & $5(3.5)$ & $19(4.2)$ & 0.276
\end{tabular}

Statistical analyses were performed using chi-squared test 
Table 5. Factors associated with unsuccessful treatment outcome in pre-XDR-TB

patients, Peru: 2011-2013

\begin{tabular}{lcccccc}
\hline Variables & \multicolumn{3}{c}{ Univariate analysis } & \multicolumn{3}{c}{ Multivariate analysis } \\
\hline & OR & Cl & p-value & OR & Cl & p-value \\
\cline { 2 - 7 } Age & 1.02 & $1.003-1.03$ & $<0.016$ & 1.01 & $0.99-1.03$ & 0.20 \\
Male sex & 1.42 & $0.97-2.1$ & $<0.071$ & 1.32 & $0.89-1.97$ & 0.17 \\
HIV+ & 0.87 & $0.32-2.29$ & 0.774 & & & \\
Diabetes & 1.64 & $0.81-3.34$ & 0.17 & 1.29 & $0.58-2.88$ & 0.53 \\
Previously treated & 2.54 & $1.73-3.74$ & $<0.001$ & 2.23 & $1.52-3.38$ & $<0.001$ \\
Pre-XDR-F & 3.07 & $1.78-5.3$ & $<0.001$ & 2.39 & $1.18-4.83$ & $<0.015$ \\
Pre-XDR-I (Km-resistant) & 0.61 & $0.41-0.89$ & $<0.012$ & 0.94 & $0.57-1.58$ & 0.84 \\
Pre-XDR-I (Cm-resistant) & 0.91 & $0.64-1.31$ & 0.613 & & & \\
Pre-XDR (Km+Cm- & 1.06 & $0.72-1.57$ & 0.77 & & & \\
resistant) & & & & & &
\end{tabular}


Figure 1. Proportion of all pre-XDR patients with positive culture according to

366 month of treatment and pre-XDR types

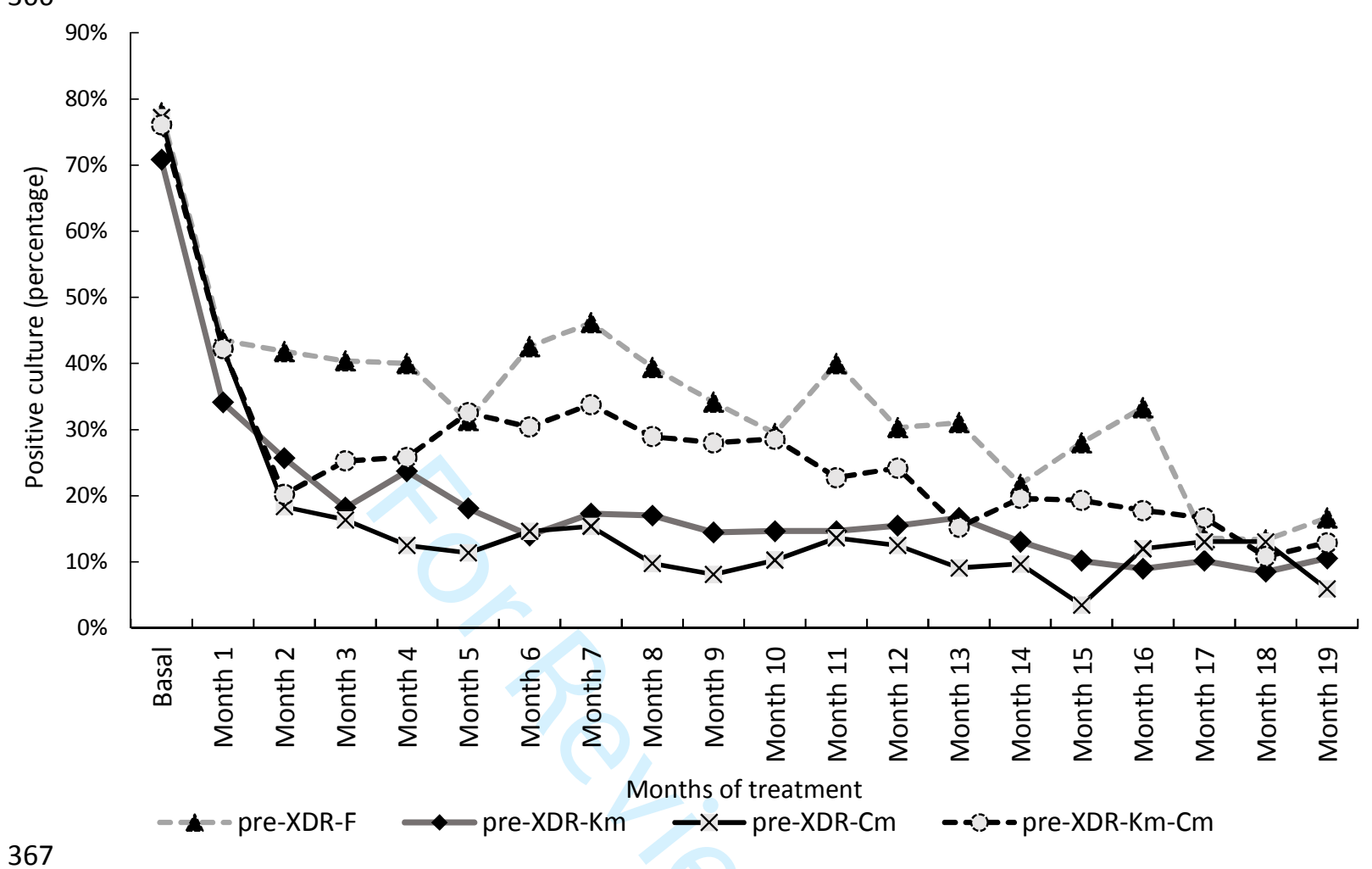




\begin{tabular}{|c|c|c|c|c|c|c|c|c|c|c|c|c|c|c|c|c|c|c|c|c|c|c|}
\hline & & Basal & Mes 1 & Mes 2 & Mes 3 & Mes 4 & Mes 5 & Mes 6 & Mes 7 & Mes 8 & Mes 9 & Mes 10 & Mes 11 & Mes 12 & Mes 13 & Mes 14 & Mes 15 & Mes 16 & Mes 17 & Mes 18 & Mes 19 & Mes 20 \\
\hline \multirow[t]{3}{*}{ pre-XDR-F } & Positivo & 81 & 27 & 23 & 21 & 18 & 16 & 20 & 18 & 13 & 14 & 10 & 14 & 10 & 9 & 5 & 7 & 7 & 3 & 2 & 2 & 2 \\
\hline & Negativo & 23 & 35 & 32 & 31 & 27 & 35 & 27 & 21 & 20 & 27 & 24 & 21 & 23 & 20 & 18 & 18 & 14 & 19 & 13 & 10 & it \\
\hline & No evalua & 16 & 58 & 65 & 68 & 75 & 69 & 73 & 81 & 87 & 79 & 86 & 85 & 87 & 91 & 97 & 95 & 99 & 98 & 105 & 108 & 110 \\
\hline \multirow[t]{3}{*}{ pre-XDR-Km } & Positivo & 131 & 44 & 28 & 20 & 23 & 19 & 14 & 14 & 16 & 13 & 11 & 11 & 11 & 11 & 9 & 6 & 6 & 6 & 4 & 4 & 3 \\
\hline & Negativo & 54 & 85 & 81 & 90 & 74 & 86 & 86 & 67 & 78 & 77 & 64 & 64 & 60 & 55 & 60 & 53 & 61 & 53 & 43 & 34 & 30 \\
\hline & No evaluado & 28 & 84 & 104 & 103 & 116 & 108 & 113 & 132 & 119 & 123 & 138 & 138 & 142 & 147 & 144 & 154 & 146 & 154 & 166 & 175 & 180 \\
\hline \multirow[t]{3}{*}{ pre-XDR-Cm } & Positivo & 71 & 28 & 11 & 9 & 6 & 5 & 6 & 6 & 4 & 3 & 4 & 3 & 4 & 3 & 3 & 1 & 3 & 3 & 3 & 1 & it \\
\hline & \begin{tabular}{|l|} 
Negativo \\
\end{tabular} & 21 & 38 & 49 & 46 & 42 & 39 & 35 & 33 & 37 & 34 & 35 & 19 & 28 & 30 & 28 & 28 & 22 & 20 & 20 & 16 & 13 \\
\hline & No evaluado & 10 & 36 & 42 & 47 & 54 & 58 & 61 & 63 & 61 & 65 & 63 & 80 & 70 & 69 & 71 & 73 & 77 & 79 & 79 & 85 & 89 \\
\hline \multirow[t]{3}{*}{ pre-XDR-Km-Cm } & Positivo & 121 & 41 & 19 & 24 & 24 & 28 & 24 & 25 & 22 & 21 & 20 & 15 & 15 & 9 & 10 & 11 & 8 & 6 & 4 & 4 & 5 \\
\hline & Negativo & 38 & 56 & 75 & 71 & 69 & 58 & 55 & 49 & 54 & 54 & 50 & 51 & 47 & 50 & 41 & 46 & 37 & 30 & 33 & 27 & 33 \\
\hline & No evaluado & 16 & 78 & 81 & 80 & 82 & 89 & 96 & 101 & 99 & 100 & 105 & 109 & 113 & 116 & 124 & 118 & 130 & 139 & 138 & 144 & 148 \\
\hline
\end{tabular}

\begin{tabular}{|c|c|c|c|c|c|c|c|c|c|c|c|c|c|c|c|c|c|c|c|c|}
\hline & & & & & & & & & & & $n t$ & & Month & onth & nth & Tonth & nth & onth & onth & Month $\left.1\right|_{M}$ \\
\hline DR-F & $78 \%$ & $44 \%$ & $42 \%$ & $40 \%$ & $40 \%$ & $31 \%$ & $43 \%$ & $46 \%$ & $39 \%$ & $34 \%$ & $29 \%$ & $40 \%$ & $30 \%$ & $31 \%$ & $22 \%$ & $28 \%$ & $33 \%$ & $14 \%$ & $13 \%$ & $17 \%$ \\
\hline re-XDR-Km & $71 \%$ & $34 \%$ & $26 \%$ & $18 \%$ & $24 \%$ & $18 \%$ & $14 \%$ & $17 \%$ & $17 \%$ & $14 \%$ & $15 \%$ & $15 \%$ & $15 \%$ & $17 \%$ & $13 \%$ & $10 \%$ & $9 \%$ & $10 \%$ & $9 \%$ & $11 \%$ \\
\hline pre-XDR-Cm & $77 \%$ & $42 \%$ & $18 \%$ & $16 \%$ & $13 \%$ & $11 \%$ & $15 \%$ & $15 \%$ & $10 \%$ & $8 \%$ & $10 \%$ & $14 \%$ & $13 \%$ & $9 \%$ & $10 \%$ & $3 \%$ & $12 \%$ & $13 \%$ & $13 \%$ & $6 \%$ \\
\hline pre-XDR-Km-Crm & $76 \%$ & $42 \%$ & $20 \%$ & $25 \%$ & $26 \%$ & $33 \%$ & $30 \%$ & $34 \%$ & $29 \%$ & $28 \%$ & $29 \%$ & $23 \%$ & $24 \%$ & $15 \%$ & $20 \%$ & $19 \%$ & $18 \%$ & $17 \%$ & $11 \%$ & $13 \%$ \\
\hline
\end{tabular}

